Gynäkol Geburtshilfliche Rundsch 1997;37:I-IV

\title{
Contents, Vol. 37, 1997
}

\section{Nr. 1}

Nr.2

\section{Editorial}

Editorial

Überiegung zur Organisation der Schwangeren- und Geburtsbetreuung

Husslein, P. (Wien)

Übersichtsarbeit

Ein präoperatives Diagnostikkonzept zur Dignitätsbeurteiiung vor laparoskopischer

Adnexchirurgie

Hesseling, M. (Wuppertal); De Wilde, R.L. (Oldenburg)

Originalarbeiten

14 Erfahrungen mit externer Qualitätssicherung im gynäkologisch-operativen Bereich

Geraedts, M.; Selbmann, H.-K. (Tubingen)

21 Fetale Ultraschallbiometrie und Fruchtwasserinsulin-konzentration bei diabetischen

Schwangeren

Hüttner, U.; Kainer, F.; Weiss, P.A.M.; Haas, J. (Graz)

25 Die Auswirkungen des Geburtsmodus auf Neugeborene mit Gastroschisis. Mit Daten des

Steirischen FehlbiIdungsregisters

Häusler, M.C.H. (Graz); Fasching, G. (Graz/Wien); Zotter, H.; Fechter, M.; Mayr, J.; Berghold, A. (Graz)

Historische Ecke

30 Befleckte Weiblichkeit - Spuren tradierter Menstruations-mythen in der Werbung für

Produkte der weiblichen Intimhygiene

Backe, J. (Würzburg)

Der klinische Fall

39 Second-look-Laparoskopie nach Tubenperforation mit dem Tuboskop

Wenzl, R.; Lehner, R.; Kiss, H.; Jirecek, S.; Huber, J.C. (Wien)

41 Spontanschwangerschaft nach Ligatur der Arteria uterina

Abendstein, B. (Hall); Brenner, E,; Klingler, P. (Innsbruck); Tscharf, J. (Hall)

Fortbildung

53 Hochschullehrertagung in Interlaken, 17.-19. April 1997

Schneider, H. (Bern)

Übersichtsarbeit

54 Neue Medikamente in der Therapie des Mammakarzinoms: Derzeitiger Stand und Ausblick Konecny, G; Untch, M.; Hepp, H. (München)

Originalarbeit 
62 Wundverschluss der Tube mittels Fibrinkleber: Endoskopische organerhaltende Therapie der Extrauteringravidität

Rudelstorfer, B.; Loidl, K.; Heider, R. (Hallein)

Der klinische Fall

68 Primäres malignes Non-Hodgkin-Lymphom der Mamma: Brusterhaltende Therapie bei 3

Patientinnen

Taus, P.; Smola, M.; Starz, 1.; Petru, E.; Höfler, G; Kronberger, L. (Graz)

Konsensus

71 Die Wirksamkeit der antenatalen Kortikosteroidtherapie auf die fetale Organreife:

Behandlungsempfehlungen der Österreichischen Gesellschaft für Prä- und Perinatale Medizin

Pollak, A.; Gruber, W.; Husslein, P. (Wien)

Gesellschaftsnachrichten

73 Österreichische Gesellschaft für Gynäkologie und Geburtshilfe (ÖGGG)

73 Stillfreundliches Krankenhaus

Winter, R. (Graz)

73 Die «Europäisierung» der Geburtshilfe und Gynäkologie

Reinold, E. (Wien)

74 European Board and College of Obstetrics and Gynaecology:The President's NewsletterNo. 1, May 1997

Beard, R.

44

1st es sinnvoll, den Eingriff nach Vecchietti pelviskopisch durchzuführen?

Borruto, F.; Fistarol, M. (Verona)

48

Erfahrung mit der Hysteroplastik. Indikationen, technische Hinweise, postoperative und

Langzeitergebnisse

Borruto, F.; Fistarol, M. (Verona)

52 Mitteilungen aus Österreich

Abstract

$\mathrm{s}$

76 XV. Akademische Tagung deutschsprechender

Hochschullehrer in der Gynäkologie und Geburtshilfe

Interlaken, 17.-19. April 1997

115 Veranstaltungskalender

KAKGER

Fax+ 41613061234 E-Mail karger@karger.ch www.karger.com

(C)1997S. KargerAG, Basel

Inhaltsverzeichnis siehe auch: www.karger.com/journals/ggr/ggrcont.htm

Nr.3

IMr.4

Editorial

117 Tötet der «lmpact Factor» die deutsche Sprache?

Haller, U. (Zurich); Hepp, H. (München); Reinold, E. (Wien) 
Übersichtsarbeit

119 Der Fetus und der Lärm

Brezinka, Ch.; Lechner, Th.; Stephan, K. (Innsbruck)

Originalarbeiten

130 Normwertkurven für intrauterine fetale Blutgas- und Säure-Basen-Parameter im 2. und 3.

Trimenon

Schröter, B.; Chaoui, R.; Glatzel, E.; Bollmann, R. (Berlin) 136 Der gedoppelte

Transpositionslappen bei der brusterhaltenden Therapie des Mammakarzinoms als vielseitige Möglichkeit der Segment- oder Quadrantektomie bei der älteren Frau: Bericht über klinische

Erfahrungen in 27 Fallen Schöndorf, N.K. (Saarlouis)

Der klinische Fall

143 Amnioninfektionssyndrom durch retroamnialen

Uterusabszess: Falsch negative bakteriologisch-diagnostische Fruchtwasserpunktion

Strauss, A.; Hasbargen, U. (München); Henckel von Donnersmark, G. (München Bogenhausen);

Westerburg, B.; Hepp, H. (München)

Spektrum

150 Der Krieg der Düfte: Bedeutung der Pheromone für die menschliche Reproduktion

Grammer, K.; Jütte, A. (Wien)

Historische Ecke

154 Der Geburtsstuhl in Texten antiker Tradition

Reuss, W.A. (Stuttgart)

161 Buchbesprechung

Mitteilungen aus Österreich

162 Symposium «Forensische Aspekte in der Geburtshilfe»

162 Die perinatalen Hirnschaden auch unterforensischen Aspekten

Schulte, F.J. (Hamburg)

169 Forensisch-gerichtsmedizinische Aspekte in der Geburtshilfe

Missliwetz, J.; Denk, W. (Wien); Gerstner, G.J. (Stockerau)

171 Medicolegal Aspects of Placental Pathology

Fox, H. (Manchester)

172 Forensische Aspekte in der Frauenheilkunde unterBerücksichtigung der Erfahrungen in der Schiedsstelle derArztekammer für Wien

Schröck, A. (Wien)

174 Gesellschaftsnachrichten 176 Veranstaltungskalender

Editorial

177 Brief des Präsidenten der ÖGGG

Salzer, H. (Wien)

Übersichtsarbeit

179 Epidemiologie und Diagnostik der zervikalen intraepithelialen Neoplasie - 1st das derzeitige

Konzept von Screening und Diagnostik noch aktuell?

Hillemanns, P.; Thaler, C; Kimmig, R. (München)

Originalarbeiten

191 Fetalblutanalyse - Pro und Contra

Roemer, V.M. (Detmold) 203 Das Schicksal des Feten mit reduziertem Fluss des Ductus venosus während der Vorhofkontraktion

Yaman, C; Arzt, W.; Aigner, M.; Tews, G. (Linz) 
209 Die Zervikalschwangerschaft. Eine gynäkologisch-geburtshilfliche Notfallsituation Pastorelli, G.; Steiner, R.; Haller, U. (Zurich)

Der klinische Fall

216 Das primäre Tubenkarzinom. Eine Einzelfalldarstellung mit Literaturübersicht

Friedrich, M.; Villena-Heinsen, C; Bonkhoff, H.; Schmidt, W. (Homburg/Saar)

Fortbildung

221 Überlebensprognose bei Ovarialkarzinomen in den Figo-Stadien III und IV: 1980-1985 versus 1986-1993.

Ein Vergleich zweier Behandlungsperioden

Knappitsch, G; Gruböck, K.; Salzer, H.; Kaider, A.; Kurz, Ch.; Vavra, N.; Speiser, P.; Sevelda, P. (Wien)

226 Buchbesprechung

Konsensus

227 Leitlinien für die operative Behandlung des Zervixkarzinoms erstellt von der

Standardkommission der Österreichischen Gesellschaft für Gynäkologie und Geburtshilfe

Winter, R. (Graz); Concin, H. (Bregenz); Fuith, L. (Eisenstadt); Lahousen, M. (Graz); Leodolter, S.; Salzer, H. (Wien); Stummvoll, W. (Linz)

Leserbrief

230 Leserbrief zu Schöndorf NK: Der gedoppelteTranspositionslappen bei der brusterhaltenden Therapie desMammakarzinoms als vielseitige Möglichkeit der Segment-oder

Quadrantektomie bei der älteren Frau: Bericht überklinische Erfahrungen in 27 Fallen

Dieterich, H. (Rheinfelden)

231 Mitteilungen aus Österreich233 Veranstaltungskalender

Autorenregister Vol. 37,1997

Sachregister Vol. 37,1997

IV

G. Gynäkol Geburtshilfliche Rundsch Vol. 37

1997

Inhaltsverzeichnis 